American Journal of Applied Sciences 9 (7): 1008-1013, 2012

ISSN 1546-9239

(C) 2012 Science Publications

\title{
Controller Based Observer in Switched System with Norm Bounded Uncertainty
}

\author{
Mongi Besbes and Elyes Maherzi \\ Department of Electrical Engineering, \\ High School of Technology and Computer Science, University of Carthage, Tunisia
}

\begin{abstract}
Problem statement: This study discusses the robust stabilization of norm bounded discrete switched systems. Approach: The proposed method is using the second Lyapunov approach and the poly-quadratic function concept. The stabilization conditions are written through linear matrix inequality relations. The control law is based on a static output feedback with the use of a switched observer. The synthesis conditions of the controller are written in the form of linear matrix inequalities difficult to resolve by current numerical solvers. That's why relaxations are proposed to mitigate the pessimism of LMI conditions obtained. Results: The poly-quadratic Lyapunov approach provides a constructive way to tackle uncertainty in the switched framework. The feasibility is illustrated by the example of discrete uncertain switched systems. Conclusion: With these results, the study of stability can be achieved for arbitrary switching laws, state-dependent, time dependent or generated by a controller. However, the implementation of the control law is possible only if the switching status is well known in real time.
\end{abstract}

Key words: Matrix inequalities, robust stabilization, arbitrary switching laws, inequality relations, systems combine continuous, stability analysis

\section{INTRODUCTION}

Many natural and artificial systems work in different operating modes, each with its own dynamic. The car changes from one dynamic mode to another with every change of speed, the human heart switches between different modes depending on the emotional state of the person. These systems concoct continuous dynamics with both synchronous or asynchronous discrete events. Such class of systems is called hybrid systems (Liberzon, 2003)

The literature has shown a growing interest in switched systems. Switched systems are hybrid systems defined by a set whose elements are dynamic continuous and/or discrete time models with commutation law which define, in time, the jumps between the elements, leading to a non stationary dynamic system. Some recent results are given in (Daafouz et al., 2001; 2002a) where a sufficient (but relatively non restrictive compared to the quadratic approach) stability condition for discrete switched systems is provided using the poly-quadratic approach recently proposed by (Daafouz and Bernussou, 2001) to analyze stability and stabilization control of Linear "Time Varying systems".

Other approaches are concerned with the determination of an act of switching between several controllers guaranteeing the stability of systems (Skafidas et al., 1999; Liberzon, 2003; Hespanha and Morse, 2002) which operating constraints require switching between multiple controllers.

This study proposes an extension of this works in the case when the switches are made between uncertain LTI systems. The control investigated is of state feedback control, observer based and dynamic controller.

\section{MATERIALS AND METHODS}

Let us consider a discrete autonomous switched system (1) where each subsystem is vitiated by a norm bounded uncertainty (Maherzi et al., 2007; Zhou and Khargonekar, 1987; 1988), this system can be described by the following equalities (1):

$\mathrm{x}(\mathrm{k}+1)=\sum_{\ell=1}^{\mathrm{M}} \xi_{\mathrm{k}}^{\ell}\left(\mathrm{A}_{\ell}+\Delta \mathrm{A}_{\ell}\right) \mathrm{x}(\mathrm{k})$

With Eq. 2 and 3:

$\Delta \mathrm{A}_{\ell}=\mathrm{D}_{\ell} \mathrm{F}_{\ell} \mathrm{E}_{\ell}$

And:

$\mathrm{F}_{\ell}^{\mathrm{T}} \mathrm{F}_{\ell} \leq \gamma_{\ell}^{2} \mathrm{I}$ 
Where:

$\ell=$ Switching index

$\mathrm{M}=$ Number of subsystems Eq. 4 and 5:

$\xi_{\mathrm{k}}^{\ell}=\left\{\begin{array}{c}1 \text { if the state matrix } \mathrm{A} \in\left(\mathrm{A}_{\ell}+\Delta \mathrm{A}_{\ell}\right) \\ 0 \text { if not }\end{array}\right.$

$\xi_{\mathrm{k}}^{\ell} \geq 0 ; \sum_{\ell=1}^{\mathrm{M}} \xi_{\mathrm{k}}^{\ell}=1 ; \xi_{\mathrm{k}}=\left[\xi_{\mathrm{k}}^{1}, \ldots, \xi_{\mathrm{k}}^{\mathrm{N}}\right]^{\mathrm{T}}$

Analysis of stability: By using Theorem developed by (Daafouz and Bernussou, 2001) the system (1) is polyquadratically stable if and only if there are $\mathrm{N}$ symmetric positive definite matrices $\mathrm{S}_{1} \ldots \mathrm{S}_{\mathrm{N}}$ and $\mathrm{N}$ symmetric positive definite matrices $G_{1} \ldots G_{N}$ matrices of appropriate dimensions satisfying Eq. 6:

$\left[\begin{array}{ll}\mathrm{G}_{\ell}+\mathrm{G}_{\ell}^{\mathrm{T}}-\mathrm{S}_{\ell} \mathrm{G}_{\ell}^{\mathrm{T}}\left(\mathrm{A}_{\ell}+\Delta \mathrm{A}_{\ell}\right)^{\mathrm{T}} \\ \left(\mathrm{A}_{\ell}+\Delta \mathrm{A}_{\ell}\right) \mathrm{G}_{\ell} & \mathrm{S}_{\mathrm{j}}\end{array}\right]>0 \forall(\ell, \mathrm{j}) \in$

$(\mathrm{e} \times \mathrm{e})$ and 8:

This is equivalent to the following inequality Eq. 7

$\left[\begin{array}{l}\mathrm{G}_{\ell}+\mathrm{G}_{\ell}^{\mathrm{T}}-\mathrm{S}_{\ell} \mathrm{G}_{\ell}^{\mathrm{T}} \mathrm{A}_{\ell}^{\mathrm{T}} \\ \mathrm{A}_{\ell} \mathrm{G}_{\ell} \\ \mathrm{S}_{\mathrm{j}}\end{array}\right]-\left[\begin{array}{cc}0 & \mathrm{G}_{\ell}^{\mathrm{T}} \widehat{\Delta \mathrm{A}} \ell^{\mathrm{T}} \\ \widehat{\Delta \mathrm{A}} \mathrm{G}_{\ell} & 0\end{array}\right]>0$

$\widehat{\Delta \mathrm{A}_{\ell}}=\mathrm{D}_{\ell}\left(-\mathrm{F}_{\ell}\right) \mathrm{E}_{\ell}$

Knowing that Eq. 9:

$\left[\begin{array}{cc}0 & \mathrm{G}_{\ell}^{\mathrm{T}} \widehat{\Delta \mathrm{A}_{\ell}^{\mathrm{T}}} \\ \widehat{\Delta \mathrm{A}_{\ell}} \mathrm{G}_{\ell} & 0\end{array}\right] \leq\left[\begin{array}{cc}\mathrm{G}_{\ell}^{\mathrm{T}} \mathrm{E}_{\ell}^{\mathrm{T}} \mathrm{E}_{\ell} \mathrm{G}_{\ell} & 0 \\ 0 & \gamma_{\ell}^{2} \mathrm{D}_{\ell} \mathrm{D}_{\ell}^{\mathrm{T}}\end{array}\right]$

So we can say that if Eq. 10:

$\left[\begin{array}{cc}\mathrm{G}_{\ell}+\mathrm{G}_{\ell}^{\mathrm{T}}-\mathrm{S}_{\ell} & \mathrm{G}_{\ell}^{\mathrm{T}} \mathrm{A}_{\ell}^{\mathrm{T}} \\ \mathrm{A}_{\ell} \mathrm{G}_{\ell} & \mathrm{Sj}\end{array}\right]-\left[\begin{array}{cc}\mathrm{G}_{\ell}^{\mathrm{T}} \mathrm{E}_{\ell}^{\mathrm{T}} \mathrm{E}_{\ell}^{\mathrm{T}} \mathrm{G}_{\ell} & 0 \\ 0 & \gamma_{\ell}^{2} \mathrm{D}_{\ell} \mathrm{D}_{\ell}^{\mathrm{T}}\end{array}\right]>0$

Then inequality (7) is true. Thereby lead to the following conditions Eq. 11:

$\left[\begin{array}{cc}\mathrm{G}_{\ell}+\mathrm{G}_{\ell}^{\mathrm{T}}-\mathrm{S}_{\ell} \mathrm{G}_{\ell}^{\mathrm{T}} \mathrm{E}_{\ell}^{\mathrm{T}} \mathrm{E}_{\ell} \mathrm{G}_{\ell} & \mathrm{G}_{\ell}^{\mathrm{T}} \mathrm{A}_{\ell}^{\mathrm{T}} \\ \mathrm{A}_{\ell} \mathrm{G}_{\ell} & \mathrm{S}_{\mathrm{j}}-\gamma_{\ell}^{2} \mathrm{D}_{\ell} \mathrm{D}_{\ell}^{\mathrm{t}}\end{array}\right]>0$

Applying the Schur complement leads to the following proposal.

Proposition 1: By using Theorem developed by (Daafouz et al., 2002b) the system described by Eq. 1 is a poly-quadratically stable "if and only if there are $\mathrm{N}$ symmetric positive definite matrices $S_{1} \ldots S_{N}$ and $N$ symmetric positive definite matrices $\mathrm{G}_{1} \ldots \mathrm{G}_{\mathrm{N}}$ matrices" of appropriate dimensions satisfying Eq. 12:

$\left[\begin{array}{cccc}\mathrm{G}_{\ell}+\mathrm{G}_{\ell}^{\mathrm{T}}-\mathrm{S}_{\ell} & \mathrm{G}_{\ell}^{\mathrm{T}} \mathrm{A}_{\ell}^{\mathrm{T}} & \mathrm{G}_{\ell}^{\mathrm{T}} \mathrm{E}_{\ell}^{\mathrm{T}} & 0 \\ \mathrm{~A}_{\ell} \mathrm{G}_{\ell} & \mathrm{S}_{\mathrm{j}} & 0 & \mathrm{D}_{\ell} \\ \mathrm{E}_{\ell} \mathrm{G}_{\ell} & 0 & \mathrm{I} & 0 \\ 0 & \mathrm{D}_{\ell}^{\mathrm{T}} & 0 & -\gamma_{\ell}^{2} \mathrm{I}\end{array}\right]$

Synthesis of dynamic control based on a switched observer: This part aims to design switched observer to reconstruct the unknown states of the system (1), these states will then be used to formulate a stabilizing control law. The ultimate goal would be to stabilize the observation error and system states (Liu and Duan, 2005).

Form of switched observer: Let us consider an observer of the form Eq. 13:

$$
\begin{aligned}
& \hat{\mathrm{x}}(\mathrm{k}+1)=\sum_{\ell=1}^{\mathrm{M}} \xi_{\ell}(\mathrm{k})\left(\begin{array}{c}
\hat{\mathrm{A}}_{\ell} \hat{\mathrm{x}}(\mathrm{k})+\mathrm{B}_{\ell} \mathrm{u}(\mathrm{k}) \\
+\mathrm{L}_{\ell}(\mathrm{y}(\mathrm{k})-\hat{\mathrm{y}}(\mathrm{k}))
\end{array}\right) \\
& \hat{\mathrm{y}}(\mathrm{k})=\sum_{\ell=1}^{\mathrm{M}} \xi_{\ell}(\mathrm{k}) \mathrm{C}_{\ell} \hat{\mathrm{x}}(\mathrm{k})
\end{aligned}
$$

The gains observation Error! Bookmark not defined. have to be calculated to guarantee the convergence to zero of the observation error Eq. 14:

$\varepsilon(k)=x(k)-\hat{x}(k)$

The evolution of the observation error is described by the following Eq. 15:

$$
\begin{aligned}
& \varepsilon(\mathrm{k}+1)=\sum_{\ell=1}^{\mathrm{M}} \xi_{\ell}(\mathrm{k})\left(\mathrm{A}_{\ell}+\Delta \mathrm{A}_{\ell}-\hat{\mathrm{A}}_{\ell}\right) \mathrm{x}(\mathrm{k}) \\
& \left.\left(\hat{\mathrm{A}}_{\ell}-\mathrm{L}_{\ell} \mathrm{C}_{\ell}\right) \varepsilon(\mathrm{k})\right)
\end{aligned}
$$

We see here that the problem of reconstruction does not make much sense, since the observation error term depends on a term such like an open loop gain on which $\mathrm{L}$ has no effect. In this term we chose to consider $\mathrm{A}_{\ell} \neq \widehat{\mathrm{A}}_{\ell}$ as well and does not constrain the $\hat{\mathrm{A}}_{\ell}$ value to have an additional degree of freedom (Vidal et al., 2002; Richard, 2003).

Control and stabilization of the system: The new augmented system is Eq. 16-22: 
Am. J. Applied Sci., 9 (7): 1008-1013, 2012

$$
\begin{aligned}
& \left(\begin{array}{c}
\mathrm{x}(\mathrm{k}+1) \\
\varepsilon(\mathrm{k}+1)
\end{array}\right)=\sum_{\ell=1}^{\mathrm{M}} \xi_{\ell}(\mathrm{k}) \phi_{\ell}\left(\begin{array}{c}
\mathrm{x}(\mathrm{k}) \\
\varepsilon(\mathrm{k})
\end{array}\right) \\
& \phi_{\ell}=\left(\begin{array}{cc}
\mathrm{A}_{\ell}+\Delta \mathrm{A}_{\ell}+\mathrm{B}_{\ell} \mathrm{k}_{\ell} & -\mathrm{B}_{\ell} \mathrm{k}_{\ell} \\
\Delta_{\ell}+\Delta \mathrm{A}_{\ell} & \hat{\mathrm{A}}_{\ell}-\mathrm{L}_{\ell} \mathrm{C}_{\ell}
\end{array}\right) \\
& \Delta_{\ell}=\mathrm{A}_{\ell}-\hat{\mathrm{A}}_{\ell}
\end{aligned}
$$

Let:

$$
\begin{aligned}
& \Phi_{\ell}^{\mathrm{T}}=\tilde{\Phi}_{\ell}+\tilde{\mathrm{D}}_{\ell} \mathrm{F}_{\ell} \tilde{\mathrm{E}}_{\ell} \\
& \tilde{\Phi}_{\ell}^{\mathrm{T}}=\left(\begin{array}{cc}
\mathrm{A}_{\ell}+\mathrm{B}_{\ell} \mathrm{K}_{\ell} & -\mathrm{B}_{\ell} \mathrm{K}_{\ell} \\
\Delta_{\ell} & \mathrm{A}_{\ell}-\Delta_{\ell}-\mathrm{L}_{\ell} \mathrm{C}_{\ell}
\end{array}\right) \\
& \tilde{\mathrm{D}}_{\ell}^{\mathrm{T}}=\left[\begin{array}{ll}
\mathrm{E}_{\ell} & 0
\end{array}\right]^{\mathrm{T}} \\
& \tilde{\mathrm{E}}_{\ell}=\left[\begin{array}{l}
\mathrm{D}_{\ell} \\
\mathrm{D}_{\ell}
\end{array}\right]^{\mathrm{T}}
\end{aligned}
$$

Using results of last proposition, system (16) is poly-quadratically stable "if and only if there are $\mathrm{N}$ symmetric positive definite matrices $S_{1} \ldots S_{N}$ and $N$ symmetric positive definite matrices $G_{1} \ldots G_{N}$ matrices" of appropriate dimensions satisfying Eq. 23:

$$
\left[\begin{array}{cccc}
\mathrm{G}_{\ell}+\mathrm{G}_{\ell}^{\tau}-\mathrm{S}_{\ell} & \mathrm{G}_{\ell}^{\tau} \tilde{\Phi}_{\ell}^{\tau} & \mathrm{G}_{\ell}^{\tau} \mathrm{E}_{\ell}^{\tau} & 0 \\
\tilde{\Phi}_{\ell} \mathrm{G}_{\ell} & \mathrm{S}_{\mathrm{i}} & 0 & \tilde{\mathrm{D}}_{\ell} \\
\tilde{\mathrm{E}}_{\ell} \mathrm{G}_{\ell} & 0 & 1 & 0 \\
0 & \tilde{\mathrm{D}}_{\ell}^{\tau} & 0 & \gamma_{\ell}^{-1} 1
\end{array}\right]>0 \forall(\ell, \mathrm{j}) \in(\mathrm{e} \times \mathrm{e})
$$

By rewriting $G_{\ell}$ as follows (24) and after the change of variables we arrive at the following stability condition (25) Eq. 24:

$$
\mathrm{G}_{\ell}=\left[\begin{array}{ll}
\mathrm{G}_{\ell, 1} & \mathrm{G}_{\ell, 2} \\
\mathrm{G}_{\ell, 3} & \mathrm{G}_{\ell, 4}
\end{array}\right]
$$

Proposition 2: The system is poly-quadratically stable "if and only if there are $\mathrm{N}$ symmetric positive definite matrices $\mathrm{S}_{1} \ldots \mathrm{S}_{\mathrm{N}}$ and $\mathrm{N}$ symmetric positive definite matrices $\mathrm{G}_{1} \ldots \mathrm{G}_{\mathrm{N}}$ matrices" of appropriate dimensions satisfying Eq. 25-29:

$$
\left[\begin{array}{ccccc}
\mathrm{G}_{\ell}+\mathrm{G}_{\ell}^{\mathrm{T}}-\mathrm{S}_{\ell} & \Gamma & \psi & 0 \\
& (.)^{\mathrm{T}} & 0 & \mathrm{I} & 0 \\
& (.)^{\mathrm{T}} & 0 & \mathrm{I} & 0 \\
0 & (.)^{\mathrm{T}} & & 0 & \gamma_{\ell}^{-2} \mathrm{I}
\end{array}\right]>0
$$

$$
\begin{aligned}
& \Gamma=\left[\begin{array}{l}
\mathrm{G}_{\ell, 1}^{\mathrm{T}}\left(\mathrm{A}_{\ell}+\mathrm{B}_{\ell} \mathrm{k}_{\ell}\right)+\mathrm{R}_{\ell}-\mathrm{G}_{\ell, 1}^{\mathrm{T}} \mathrm{B}_{\ell} \mathrm{k}_{\ell}+\mathrm{G}_{\ell, 3}^{\mathrm{T}} \mathrm{A}_{\ell}-\mathrm{R}_{\ell}-\mathrm{U}_{\ell} \mathrm{C}_{\ell} \\
\mathrm{G}_{\ell, 2}^{\mathrm{T}}\left(\mathrm{A}_{\ell}+\mathrm{B}_{\ell} \mathrm{k}_{\ell}\right)+\mathrm{F}_{\ell}-\mathrm{G}_{\ell, 2}^{\mathrm{T}} \mathrm{B}_{\ell} \mathrm{k}_{\ell}+\mathrm{G}_{\ell, 4}^{\mathrm{T}} \mathrm{A}_{\ell}-\mathrm{F}_{\ell}-\mathrm{V}_{\ell} \mathrm{C}_{\ell}
\end{array}\right] \\
& \psi\left[\begin{array}{l}
\mathrm{G}_{\ell, 1}^{\mathrm{T}} \mathrm{D}_{\ell}+\mathrm{G}_{\ell, 3}^{\mathrm{T}} \mathrm{D}_{\ell} \\
\mathrm{G}_{\ell, 2}^{\mathrm{T}} \mathrm{D}_{\ell}+\mathrm{G}_{\ell, 4}^{\mathrm{T}} \mathrm{D}_{\ell}
\end{array}\right] \\
& {\left[\begin{array}{c}
\mathrm{R}_{\ell} \\
\mathrm{F}_{\ell}
\end{array}\right]=\left[\begin{array}{l}
\mathrm{G}_{\ell, 3}^{\mathrm{T}} \\
\mathrm{G}_{\ell, 4}^{\mathrm{T}}
\end{array}\right] \mathrm{L}_{\ell}} \\
& {\left[\begin{array}{c}
\mathrm{U}_{\ell} \\
\mathrm{V}_{\ell}
\end{array}\right]=\left[\begin{array}{l}
\mathrm{G}_{\ell, 3}^{\mathrm{T}} \\
\mathrm{G}_{\ell, 4}^{\mathrm{T}}
\end{array}\right] \Delta_{\ell}}
\end{aligned}
$$

Find a solution to the LMIs (26) while respecting the equality constraints (28) and (29) is a non convex problem a solution exists only if the following rank conditions are verified Eq. 30:

$$
\operatorname{rank}\left[\begin{array}{c}
\mathrm{G}_{\ell, 3}^{\mathrm{T}} \\
\mathrm{G}_{\ell, 4}^{\mathrm{T}}
\end{array}\right]=\operatorname{rank}\left[\begin{array}{cc}
\mathrm{U}_{\ell} & \mathrm{G}_{\ell, 3}^{\mathrm{T}} \\
\mathrm{V}_{\ell} & \mathrm{G}_{\ell, 4}^{\mathrm{T}}
\end{array}\right]=\operatorname{rank}\left[\begin{array}{cc}
\mathrm{R}_{\ell} & \mathrm{G}_{\ell, 3}^{\mathrm{T}} \\
\mathrm{F}_{\ell} & \mathrm{G}_{\ell, 4}^{\mathrm{T}}
\end{array}\right]
$$

So to find solutions to the LMIs (25) that satisfy the constraints (28) and (29) it is possible to apply an iterative algorithm of solving the LMI (25) iteratively by testing the rank condition (28) and (29) at each stage (Halabi, 2005).

Another approach would be to fix.

$\mathrm{G}_{\ell, 3}=0$ then the condition (25) becomes Eq. 31-36:

$$
\left[\begin{array}{llll}
\mathrm{G}_{\ell}+\mathrm{G}_{\ell}^{\mathrm{T}}-\mathrm{S}_{\ell} & \Gamma_{3} & \psi_{3} & 0 \\
(.)^{\mathrm{T}} & \mathrm{S}_{\mathrm{j}} & 0 & \mathrm{E}_{\ell} \\
(.)^{\mathrm{T}} & 0 & \mathrm{I} & 0 \\
0 & & (.)^{\mathrm{T}} 0 & \gamma_{\ell}^{-2} \mathrm{I}
\end{array}\right]>0
$$

$\Gamma_{3}=\left[\begin{array}{l}\mathrm{G}_{\ell, 1}^{\mathrm{T}}\left(\mathrm{A}_{\ell}+\mathrm{B}_{\ell} \mathrm{k}_{\ell}\right)+\mathrm{R}_{\ell}-\mathrm{G}_{\ell, 1}^{\mathrm{t}} \mathrm{b}_{\ell} \mathrm{K}_{\ell} \\ \mathrm{G}_{\ell, 2}^{\mathrm{T}}\left(\mathrm{A}_{\ell}+\mathrm{B}_{\ell} \mathrm{k}_{\ell}\right)+\mathrm{F}_{\ell}-\mathrm{G}_{\ell, 2}^{\mathrm{T}} \mathrm{B}_{\ell} \mathrm{k}_{\ell} \\ +\mathrm{G}_{\ell, 4}^{\mathrm{T}} \mathrm{A}_{\ell}-\mathrm{F}_{\ell}-\mathrm{V}_{\ell} \mathrm{C}_{\ell}\end{array}\right]$

$\psi_{3}=\left[\begin{array}{l}\mathrm{G}_{\ell, 1}^{\mathrm{T}} \mathrm{D}_{\ell} \\ \mathrm{G}_{\ell, 2}^{\mathrm{T}} \mathrm{D}_{\ell}+\mathrm{G}_{\ell, 4}^{\mathrm{T}} \mathrm{D}_{\ell}\end{array}\right]$

$\hat{\mathrm{A}}_{\ell}=\mathrm{A}_{\ell}-\Delta_{\ell}$

$\Delta_{\ell}=\left(\mathrm{G}_{\ell, 4}^{\mathrm{T}}\right)^{-1} \mathrm{~F}_{\ell}$

$\mathrm{L}_{\ell}=\mathrm{G}_{\ell, 4}^{\mathrm{T}} \mathrm{V}_{\ell}$ 
$\mathrm{G}_{\ell, 3}=\mathrm{G}_{\ell, 4}$ the condition (25) becomes Eq. 37-42:

$\left[\begin{array}{cccc}\mathrm{G}_{\ell}+\mathrm{G}_{\ell}^{\mathrm{T}}-\mathrm{S}_{\ell} & \Gamma_{34} & \Psi_{34} & 0 \\ (.)^{\mathrm{T}} & \mathrm{S}_{\mathrm{j}} & 0 & \mathrm{E}_{\ell} \\ (.)^{\mathrm{T}} & 0 & \mathrm{I} & 0 \\ 0 & (.)^{\mathrm{T}} & 0 & \gamma_{\ell}^{-2} \mathrm{I}\end{array}\right]>0$

$\phi_{34}=\left[\begin{array}{l}\mathrm{G}_{\ell, 1}^{\mathrm{T}}\left(\mathrm{A}_{\ell}+\mathrm{B}_{\ell} \mathrm{k}_{\ell}\right)+\mathrm{F}_{\ell}-\mathrm{G}_{\ell, 1}^{\mathrm{T}} \mathrm{B}_{\ell} \mathrm{k}_{\ell}+\mathrm{G}_{\ell, 4}^{\mathrm{T}} \mathrm{A}_{\ell}-\mathrm{F}_{\ell}-\mathrm{V}_{\ell} \mathrm{C}_{\ell} \\ \mathrm{G}_{\ell, 2}^{\mathrm{T}}\left(\mathrm{A}_{\ell}+\mathrm{B}_{\ell} \mathrm{k}_{\ell}\right)+\mathrm{F}_{\ell}-\mathrm{G}_{\ell, 2}^{\mathrm{T}} \mathrm{B}_{\ell} \mathrm{k}_{\ell}+\mathrm{G}_{\ell, 4}^{\mathrm{T}} \mathrm{A}_{\ell}-\mathrm{F}_{\ell}-\mathrm{V}_{\ell} \mathrm{C}_{\ell}\end{array}\right]$

$\Psi_{34}=\left[\begin{array}{l}\mathrm{G}_{\ell, 1}^{\mathrm{T}} \mathrm{D}_{\ell}+\mathrm{G}_{\ell, 4}^{\mathrm{T}} \mathrm{D}_{\ell} \\ \mathrm{G}_{\ell, 2}^{\mathrm{T}} \mathrm{D}_{\ell}+\mathrm{G}_{\ell, 4}^{\mathrm{T}} \mathrm{D}_{\ell}\end{array}\right]$

With:

$\hat{\mathrm{A}}_{\ell}=\mathrm{A}_{\ell}-\Delta_{\ell}$

$\Delta_{\ell}=\left(\mathrm{G}_{\ell, 4}\right)^{-1} \mathrm{~F}_{\ell}$

$\mathrm{L}_{\ell}=\mathrm{G}_{\ell, 4}^{\mathrm{T}} \mathrm{V}_{\ell}$

\section{RESULTS}

It is clear that these methods are conservative, because the control gains and observers are calculated separately and we imposed a special structure in the matrix variable G. However these methods are interesting because they allow a relaxation of conditions LMIs to be solved by the freedom granted to the matrices $\hat{\mathrm{A}}_{\ell}$ required for the construction of the observers.

Illustration example: It' is a benchmark example, commonly used in the literature, which we added an uncertainty of norm bounded type.

$$
\begin{aligned}
X(k+1) & =\left[\begin{array}{l}
\mathrm{x}_{1}(\mathrm{k}+1) \\
\mathrm{x}_{2}(\mathrm{k}+1) \\
\mathrm{x}_{3}(\mathrm{k}+1)
\end{array}\right] \\
& +\mathrm{AX}(\mathrm{k})+\mathrm{Bu}(\mathrm{k}) \mathrm{y}(\mathrm{k})=\left[\mathrm{x}_{1}(\mathrm{k}) \mathrm{x}_{2}(\mathrm{k})\right]^{\mathrm{T}} \\
\mathrm{A} & =(\mathrm{I}+\mathrm{aI})\left[\begin{array}{lll}
0.2113 & 0.0087 & 0.4524 \\
0.0824 & 0.8096 & 0.8075 \\
0.7599 & 0.8474 & 0.4832
\end{array}\right] \\
\mathrm{B} & =\left[\begin{array}{ll}
0.6135 & 0.6538 \\
0.2749 & 0.4899 \\
0.8807 & 0.7741
\end{array}\right]
\end{aligned}
$$

One of the two actuators can fail, in this case the matrix B may take three different values:

$$
\begin{aligned}
& \mathrm{B}_{1}=\left[\begin{array}{ll}
0.6135 & 0.6538 \\
0.2749 & 0.4899 \\
0.8807 & 0.7741
\end{array}\right] \\
& \mathrm{B}_{2}=\left[\begin{array}{ll}
0.6135 & 0.0000 \\
0.2749 & 0.0000 \\
0.8807 & 0.0000
\end{array}\right] \\
& \mathrm{B}_{2}=\left[\begin{array}{ll}
0.000 & 0.6538 \\
0.000 & 0.4899 \\
0.000 & 0.7741
\end{array}\right]
\end{aligned}
$$

A representation norm bounded uncertainty in the dynamical matrix can be given by:

$$
\Delta \mathrm{A}_{\ell}=\mathrm{DFE} ; \mathrm{D}_{\ell}=\mathrm{A}_{\ell} ; \mathrm{F}^{\mathrm{T}} \mathrm{F} \leq \alpha^{2} \mathrm{I} ; \mathrm{E}=\mathrm{I}
$$

For a maximum value of $=0.18$, the proposition 1 , allows us to obtain the state feedback gains by replacing $\mathrm{A}_{\ell}$ by:

$$
\begin{aligned}
\mathrm{A}_{\ell} & +\mathrm{B}_{\ell} \mathrm{K}_{\ell}: \\
\mathrm{k}_{1} & =\left[\begin{array}{lll}
-1.4882 & 0.7063 & 1.7174 \\
0.7982 & -1.6053 & -2.5123
\end{array}\right] \\
\mathrm{k}_{2} & =\left[\begin{array}{ccc}
-0.6604 & -0.9712 & -2.5123 \\
0.00000 & 0.00000 & 0.00000
\end{array}\right] \\
\mathrm{k}_{2} & =\left[\begin{array}{llr}
-0.6604 & -0.9712 & -2.5123 \\
0.00000 & 0.00000 & 0.00000
\end{array}\right]
\end{aligned}
$$

Based on the condition (5.47) we obtain the following observers:

$$
\begin{aligned}
\hat{\mathrm{A}}_{1} & =\left[\begin{array}{lll}
0.2203 & 0.0207 & 0.4472 \\
0.1076 & 0.8584 & 0.8031 \\
0.7844 & 0.9053 & 0.4856
\end{array}\right] \\
\mathrm{L}_{1} & =\left[\begin{array}{ll}
0.3740 & 0.5572 \\
0.4396 & 1.9716 \\
1.0580 & 1.7440
\end{array}\right] \\
\hat{\mathrm{A}}_{2} & =\left[\begin{array}{lll}
0.2126 & 0.0383 & 0.4729 \\
0.0876 & 0.9036 & 0.8694 \\
0.7692 & 0.9396 & 0.5361
\end{array}\right]
\end{aligned}
$$




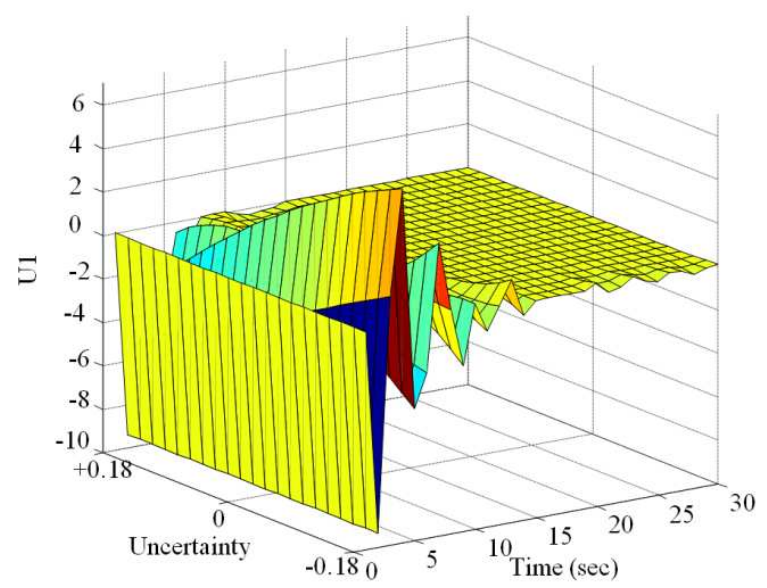

Fig. 1: Evolution of actuator 1

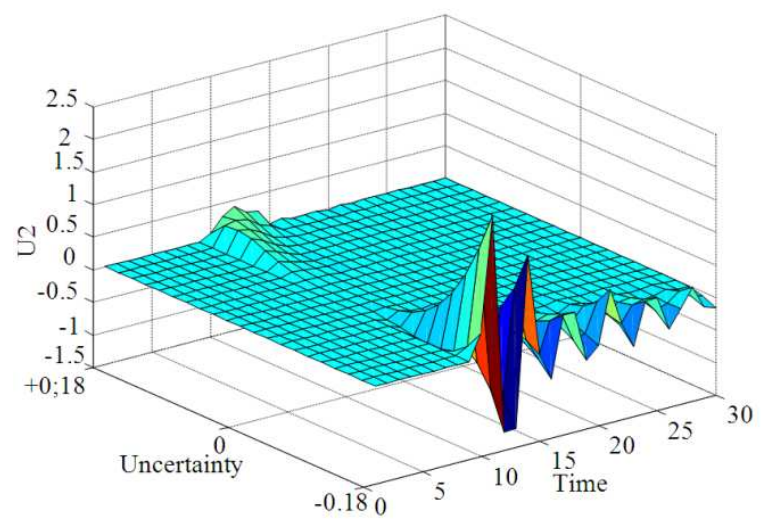

Fig. 2: Evolution of actuator 2

$$
\begin{aligned}
L_{2} & =\left[\begin{array}{ll}
0.3754 & 0.5604 \\
0.4450 & 1.9813 \\
1.0628 & 1.7525
\end{array}\right] \\
\hat{\mathrm{A}}_{3} & =\left[\begin{array}{lll}
0.2252 & 0.8499 & 0.4597 \\
0.0677 & 0.8499 & 0.8359 \\
0.7555 & 0.8953 & 0.5076
\end{array}\right] \\
\mathrm{L}_{3} & =\left[\begin{array}{ll}
0.3548 & 0.5186 \\
0.3882 & 1.8695 \\
1.0127 & 1.6541
\end{array}\right]
\end{aligned}
$$

The conditions obtained from the formulation norm bounded uncertainties have the advantage of reducing the number of LMIs to be solved compared to the polytopical formulation of uncertainty which, admittedly, is less conservative but generates much of LMIs to solve.

Figure 1 and 2 shows the evolution of controls $u_{1}$ and $\mathrm{u}_{2}$ for the uncertainty range with a loss of control $\mathrm{u} 1$ [15s 20s] and loss of control u2 between times [0s 10s].

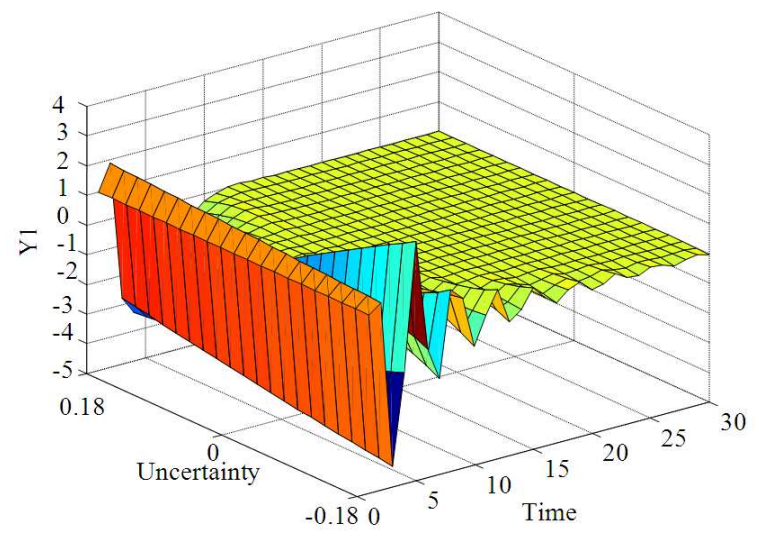

Fig. 3: System output $Y_{1}$

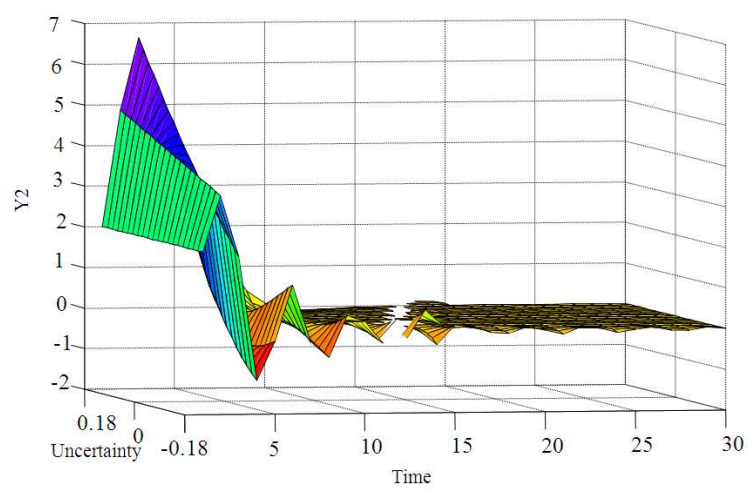

Fig. 4: System output $Y_{2}$

Figure 3 and 4 show the system stability despite uncertainties and actuator failures.

\section{DISCUSSION}

The poly-quadratic Lyapunov approach provides a constructive way to tackle bounded uncertainty in the switched framework. The controller synthesis conditions are dependent on information provided by observers and the feasibility of LMIs generated by the calculations. We believe that the proposed conditions are not pessimistic and against this approach may offer alternatives to conventional methods of synthesis of current regulator for this particular type of system.

\section{CONCLUSION}

With these results, the study of stability can be achieved for arbitrary switching laws, state-dependent, time dependent or generated by a controller. However, the implementation of the control law is possible only if switching law status is known in real time (Gao et al., 2004; Hetel et al., 2008). 
In terms of outlook, an important point, to seek, is to develop conditions based even on partial knowledge of the switching law without necessarily having a thorough knowledge of real-time switching.

Information obtained in advance of the switching law can afford to give less restrictive conditions, not having to account for any switching law possible. Indeed, it is possible in some practical applications, such as control systems via computer networks, to estimate an interval containing the delay as a function of various parameters of the network. It will be interesting, in this case, to define conditions of stabilization not only taking into account the parameter uncertainties but also uncertainties about the delay.

\section{REFERENCES}

Daafouz, J. and J. Bernussou, 2001. Parameter dependent Lyapunov functions for discrete time systems with time varying parametric uncertainties. Syst. Control Lett., 43: 355-359. DOI: 10.1016/S0167-6911(01)00118-9

Daafouz, J., P. Riedinger and C. Lung, 2002a. Stability analysis and control synthesis for switched systems: A switched Lyapunov function approach. IEEE Trans. Automatic Control, 47: 1883-1887. DOI: 10.1109/TAC.2002.804474

Daafouz, J., G. Millerioux and C. Lung, 2002b. A polyquadratic stability based approach for linear switched systems. Int. J. Control, 75: 1302-1310. DOI: $10.1080 / 0020717021000023735$

Daafouz, J., P. Riedinger and I. Claude, 2001. Static output feedback control for switched systems. Proceedings of the 40th IEEE Conference on Decision and Control, Dec. 4-7, IEEE Xplore Press, Orlando, Florida USA., pp: 2093-2094. DOI: 10.1109/. 2001.980560

Gao, H., J. Lam, C. Wang and Y. Wang, 2004. Delaydependent output-feedback stabilization of discrete-time systems with time-varying state delay. IEE Proc. Control Theory Appli., 151: 691698. DOI : 10.1049/ip-cta:20040822

Halabi, S., 2005. Filtrage robuste pour les systemes stochastiques incertains. Thèse de doctorat, Université Henri Poincarré Nancy.
Hespanha, J.P. and A.S. Morse, 2002. Switching between stabilizing controllers. Automatic, 38: 1905-1917. DOI: 10.1016/S0005-1098(02)00139-5

Hetel, L., J. Daafouz and C. Iung, 2008. Equivalence between the Lyapunov-Krasovskii functionals approach for discrete delay systems and that of the stability conditions for switched systems. Nonlinear Anal.: Hybrid Syst., 2: 697-705. DOI: 10.1016/j. Nash. 2007.11.003

Liberzon, D., 2003. Switching in Systems and Control. 1st Edn., Springer, Boston, ISBN-10: 0817642978, pp: 233.

Liu, X.L. and G.R. Duan, 2005. Robust stabilization of switched systems with input saturation. Proceedings of the 2005 International Conference on Machine Learning and Cybernetics, Aug. 18-21, IEEE Xplore Press, Guangzhou, China, pp: 10211025. DOI: 10.1109/ICMLC.2005.1527093

Maherzi, E., J. Bernussou and R. Mhiri, 2007. Stability and stabilization of uncertain switched systems, a polyquadratic Lyapunov approach. Int. J. Sci. Techniques Automatic Control, 1 : 61-74.

Richard, J.P., 2003. Time-delay systems: An overview of some recent advances and open problems. Automatic, 39: 1667-1694. DOI: 10.1016/S00051098(03)00167-5

Skafidas, E., R.J. Evans, A.V. Savkin and I.R. Petersen, 1999. Stability results for switched controller systems. Automatic, 35: 553-564. DOI: 10.1016/S0005-1098(98)00167-8

Vidal, R., A. Chiuso and S. Soatto, 2002. Observability and identifiability of jump linear systems. Proceedings of 41st IEEE Conference on Decision and Control, Dec. 10-13, IEEE Xplore Press, Las Vegas NV, USA., pp: 3614-3619. DOI: 10.1109/CDC.2002.1184923

Zhou, K. and P. Khargonekar, 1987. Stability robustness bounds for linear state-space models with structured uncertainty. IEEE Trans. Automatic Control, 32: 621-623. DOI: 10.1109/TAC.1987.1104667

Zhou, K. and P.P. Khargonekar, 1988. Robust stabilization of linear systems with norm-bounded time-varying uncertainty. Syst. Control Lett., 10: 17-20. DOI: 10.1016/0167-6911 (88)90034-5 